\title{
Lie Algebra of Unit Tangent Bundle in Minkowski 3-Space
}

\author{
Murat Bekar*
}

(Communicated by Levent Kula )

\begin{abstract}
In this paper, a one-to-one correspondence between the set of unit split semi-quaternions and unit tangent bundle of semi-Euclidean plane is given. It is shown that the set of unit split semiquaternions based on the group operation of multiplication is a Lie group. The Lie algebra of this group, consisting of the vector space matrix of the angular velocity vectors, is also considered. Planar rotations in Euclidean plane are expressed using split semi-quaternions. Some examples are given to illustrate the findings.
\end{abstract}

\section{Introduction}

Real-quaternions were first described by Irish mathematician Sir William Rowan Hamilton in 1843. The space of real-quaternions coincides with the 4-dimensional vector space over the real-numbers. Quaternions provide a simple and elegant way especially for describing rotations and reflections in space, and therefore have wide applications in Engineering. Moreover, they can be used alongside other methods, such as Euler angles, matrices and mechanics.

There is a one-to-one correspondence between the set of unit semi-quaternions and the unit tangent bundle of Euclidean plane $\mathbb{R}^{2}$, see [8]. The aim of this paper is to generalize the idea of the paper [8] to Minkowski 3-space $\mathbb{R}_{1}^{3}$ by constructing an isomorphism between the unit tangent bundle of semi-Euclidean plane $\mathbb{R}_{1}^{2}$ and the unit split semi 3-sphere $T \mathbb{R}_{1}^{2}$. It is shown that $T \mathbb{R}_{1}^{2}$ is a Lie group and its Lie algebra is non-compact. As a geometric comment, planar rotations in Euclidean plane $\mathbb{R}^{2}$ are expressed in terms of split semi-quaternions and some examples are given to illustrate the main results.

\section{Preliminaries}

In this section, definitions of the algebras real-quaternions and split semi-quaternions are given. Then, some basic properties of these algebras are briefly introduced. To distinguish real-quaternions from split semi-quaternions, a real-quaternion is expressed by $q=q_{0}+q_{1} \boldsymbol{i}+q_{2} \boldsymbol{j}+q_{3} \boldsymbol{k}$ and a split semi-quaternion by $\mathrm{q}=\mathrm{q}_{0}+\mathrm{q}_{1} \mathbf{i}+\mathrm{q}_{2} \mathbf{j}+\mathrm{q}_{3} \mathbf{k}$.

\subsection{Real-Quaternions}

The real algebra of real-quaternions, denoted by $\mathbb{H}$, is in one-to-one correspondence with the linear Euclidean space $\mathbb{R}^{4}$ having a standard basis $\{1, \boldsymbol{i}, \boldsymbol{j}, \boldsymbol{k}\}$. So, a real-quaternion $q$ exists from a scalar part and three imaginary parts:

$$
q=q_{0}+q_{1} \boldsymbol{i}+q_{2} \boldsymbol{j}+q_{3} \boldsymbol{k}
$$


where $q_{i} \in \mathbb{R}(i=0, \ldots, 3)$ and $\boldsymbol{i}, \boldsymbol{j}, \boldsymbol{k}$ are the imaginary units. $S_{q}=q_{0} \in \mathbb{R}$ is called the scalar part and $\mathbf{V}_{q}=$ $q_{1} \boldsymbol{i}+q_{2} \boldsymbol{j}+q_{3} \boldsymbol{k}$ the vector part. The multiplication rules between the imaginary units $\boldsymbol{i}, \boldsymbol{j}, \boldsymbol{k}$ are:

$$
\begin{gathered}
\boldsymbol{i}^{2}=\boldsymbol{j}^{2}=\boldsymbol{k}^{2}=\boldsymbol{i} \boldsymbol{j} \boldsymbol{k}=-1, \\
\boldsymbol{i} \boldsymbol{j}=-\boldsymbol{j} \boldsymbol{i}=\boldsymbol{k}, \quad \boldsymbol{j} \boldsymbol{k}=-\boldsymbol{k} \boldsymbol{j}=\boldsymbol{i}, \quad \boldsymbol{k} \boldsymbol{i}=-\boldsymbol{i} \boldsymbol{k}=\boldsymbol{j} .
\end{gathered}
$$

Thus, the multiplication of real-quaternions is not commutative. If $S_{q}=0$, then $q$ is said to be a pure and is denoted by $\boldsymbol{q}$.

The conjugate of a real-quaternion $q=q_{0}+q_{1} \boldsymbol{i}+q_{2} \boldsymbol{j}+q_{3} \boldsymbol{k}=S_{q}+\mathbf{V}_{q}$ is

$$
\bar{q}=q_{0}-q_{1} \boldsymbol{i}-q_{2} \boldsymbol{j}-q_{3} \boldsymbol{k}=S_{q}-\mathbf{V}_{q},
$$

see $[3,4,5,2]$.

In the scalar-vector representation, multiplication of the real-quaternions $q=S_{q}+\mathbf{V}_{q}$ and $p=S_{p}+\mathbf{V}_{p}$ is

$$
q p=S_{q} S_{p}-\left\langle\mathbf{V}_{q}, \mathbf{V}_{p}\right\rangle+S_{q} \mathbf{V}_{p}+S_{p} \mathbf{V}_{q}+\mathbf{V}_{q} \times \mathbf{V}_{p},
$$

where $\left\langle\mathbf{V}_{q}, \mathbf{V}_{p}\right\rangle$ and $\mathbf{V}_{q} \times \mathbf{V}_{p}$ are, respectively, the usual inner and vector products of $\mathbf{V}_{q}$ and $\mathbf{V}_{p}$ in $\mathbb{R}^{3}$. In the real quantities $q_{i} \in \mathbb{R}$ and $p_{i} \in \mathbb{R}(i=0, \ldots, 3)$ representation, the multiplication of $q=q_{0}+q_{1} \boldsymbol{i}+q_{2} \boldsymbol{j}+q_{3} \boldsymbol{k}$ and $p=p_{0}+p_{1} \boldsymbol{i}+p_{2} \boldsymbol{j}+p_{3} \boldsymbol{k}$ can be given also by the matrix product

$$
q p:=\left(\begin{array}{rrrr}
q_{0} & -q_{1} & -q_{2} & -q_{3} \\
q_{1} & q_{0} & -q_{3} & q_{2} \\
q_{2} & q_{3} & q_{0} & -q_{1} \\
q_{3} & -q_{2} & q_{1} & q_{0}
\end{array}\right)\left(\begin{array}{l}
p_{0} \\
p_{1} \\
p_{2} \\
p_{3}
\end{array}\right) .
$$

The norm of $q=q_{0}+q_{1} \boldsymbol{i}+q_{2} \boldsymbol{j}+q_{3} \boldsymbol{k}$ is

$$
N_{q}=q \bar{q}=\bar{q} q=q_{0}{ }^{2}+q_{1}{ }^{2}+{q_{2}}^{2}+q_{3}{ }^{2}
$$

and its modulus is

$$
\|q\|=\sqrt{N_{q}}=\sqrt{q_{0}^{2}+q_{1}^{2}+q_{2}^{2}+q_{3}^{2}} .
$$

Real-quaternions with $N_{q}=1$ are called unit.

The multiplicative-inverse of a non-zero real-quaternion $q=q_{0}+q_{1} \boldsymbol{i}+q_{2} \boldsymbol{j}+q_{3} \boldsymbol{k}$ is

$$
q^{-1}=\frac{\bar{q}}{N_{q}} .
$$

Hence the real-quaternion algebra $\mathbb{H}$ forms a division algebra. For a unit real-quaternion $q$, we have $q^{-1}=\bar{q}$. For more information about real-quaternions see $[11,12,14,17,15,6]$.

The division algebra of real-quaternions $\mathbb{H}$ is isomorphic to the Clifford algebra $C \ell_{0,2}$ spanned by the basis $\left\{1, e_{1}, e_{2}, e_{1} e_{2}\right\}$ in 2-dimension by identifying the quaternionic units $\boldsymbol{i}, \boldsymbol{j}, \boldsymbol{k}$ with $e_{1}, e_{2}, e_{1} e_{2}=e_{3}$ in $C \ell_{0,2}$, respectively. The standard anti-commuting orthonormal basis elements $e_{1}, e_{2}$ satisfy

$$
\left(e_{1}\right)^{2}=\left(e_{2}\right)^{2}=\left(e_{3}\right)^{2}=-1 \quad \text { and } \quad e_{1} e_{2}=-e_{2} e_{1},
$$

see [1].

\subsection{Split Semi-Quaternions and Unit Tangent Bundle of $\mathbb{R}_{1}^{2}$}

Like real-quaternions a split semi-quaternion q exists from a scalar part and three imaginary parts:

$$
\mathrm{q}=\mathrm{q}_{0}+\mathrm{q}_{1} \mathbf{i}+\mathrm{q}_{2} \mathbf{j}+\mathrm{q}_{3} \mathbf{k},
$$

where $\mathrm{q}_{i} \in \mathbb{R}(i=0, \ldots, 3)$ and $\mathbf{i}, \mathbf{j}, \mathbf{k}$ are the imaginary units. $S_{\mathrm{q}}=\mathrm{q}_{0} \in \mathbb{R}$ is called the scalar part and $\mathbf{V}_{\mathrm{q}}=$ $\mathrm{q}_{1} \mathbf{i}+\mathrm{q}_{2} \mathbf{j}+\mathrm{q}_{3} \mathbf{k}$ the vector part. The multiplication rules between the imaginary units $\mathbf{i}, \mathbf{j}, \mathbf{k}$ are:

$$
\begin{gathered}
\mathbf{i}^{2}=+1, \quad \mathbf{j}^{2}=\mathbf{k}^{2}=0, \\
\mathbf{i j}=-\mathbf{j i}=\mathbf{k}, \quad \mathbf{j} \mathbf{k}=-\mathbf{k} \mathbf{j}=0, \quad \mathbf{k} \mathbf{i}=-\mathbf{i k}=-\mathbf{j} .
\end{gathered}
$$


Like real-quaternions, the multiplication of split semi-quaternions is not commutative. We denote the algebra of split semi-quaternions by $\mathbb{H}_{\mathrm{S}}$. If $S_{\mathrm{q}}=0$, then q is said to be a pure and is denoted by $\mathbf{q}$.

The conjugate of a split semi-quaternion $\mathrm{q}=\mathrm{q}_{0}+\mathrm{q}_{1} \mathbf{i}+\mathrm{q}_{2} \mathbf{j}+\mathrm{q}_{3} \mathbf{k}=S_{\mathrm{q}}+\mathbf{V}_{\mathrm{q}}$ is

$$
\overline{\mathrm{q}}=S_{\mathrm{q}}-\mathbf{V}_{\mathrm{q}} .
$$
is

In the scalar-vector representation, multiplication of the split semi-quaternions $\mathrm{q}=S_{\mathrm{q}}+\mathbf{V}_{\mathrm{q}}$ and $\mathrm{p}=S_{\mathrm{p}}+\mathbf{V}_{\mathrm{p}}$

$$
\mathrm{qp}=S_{\mathrm{q}} S_{\mathrm{p}}-\left\langle\mathbf{V}_{\mathrm{q}}, \mathbf{V}_{\mathrm{p}}\right\rangle^{*}+S_{\mathrm{q}} \mathbf{V}_{\mathrm{p}}+S_{\mathrm{p}} \mathbf{V}_{\mathrm{q}}+\mathbf{V}_{\mathrm{q}} \times{ }^{*} \mathbf{V}_{\mathrm{p}},
$$

where $\left\langle\mathbf{V}_{\mathrm{q}}, \mathbf{V}_{\mathrm{p}}\right\rangle^{*}=-\mathrm{q}_{1} \mathrm{p}_{1}$ is the inner product and $\mathbf{V}_{\mathrm{q}} \times^{*} \mathbf{V}_{\mathrm{p}}=0 \mathbf{i}-\left(\mathrm{q}_{3} \mathrm{p}_{1}-\mathrm{q}_{1} \mathrm{p}_{3}\right) \mathbf{j}+\left(\mathrm{q}_{1} \mathrm{p}_{2}-\mathrm{q}_{2} \mathrm{p}_{1}\right) \mathbf{k}$ the vector product.

In the real quantities $\mathrm{q}_{i} \in \mathbb{R}$ and $\mathrm{p}_{i} \in \mathbb{R}(i=0, \ldots, 3)$ representation, the multiplication of the split semiquaternions $\mathrm{q}=\mathrm{q}_{0}+\mathrm{q}_{1} \mathbf{i}+\mathrm{q}_{2} \mathbf{j}+\mathrm{q}_{3} \mathbf{k}$ and $\mathrm{p}=\mathrm{p}_{0}+\mathrm{p}_{1} \mathbf{i}+\mathrm{p}_{2} \mathbf{j}+\mathrm{p}_{3} \mathbf{k}$ can be given also by the matrix product

$$
q p:=\left(\begin{array}{rrrr}
\mathrm{q}_{0} & \mathrm{q}_{1} & 0 & 0 \\
\mathrm{q}_{1} & \mathrm{q}_{0} & 0 & 0 \\
\mathrm{q}_{2} & -\mathrm{q}_{3} & \mathrm{q}_{0} & \mathrm{q}_{1} \\
\mathrm{q}_{3} & -\mathrm{q}_{2} & \mathrm{q}_{1} & \mathrm{q}_{0}
\end{array}\right)\left(\begin{array}{l}
\mathrm{p}_{0} \\
\mathrm{p}_{1} \\
\mathrm{p}_{2} \\
\mathrm{p}_{3}
\end{array}\right) .
$$

And thus, the matrix representation of a split semi-quaternion $r=r_{0}+r_{1} \mathbf{i}+r_{2} \mathbf{j}+r_{3} \mathbf{k}$ can be given as

$$
\mathrm{R}:=\left(\begin{array}{cccc}
\mathrm{r}_{0} & \mathrm{r}_{1} & 0 & 0 \\
\mathrm{r}_{1} & \mathrm{r}_{0} & 0 & 0 \\
\mathrm{r}_{2} & -\mathrm{r}_{3} & \mathrm{r}_{0} & \mathrm{r}_{1} \\
\mathrm{r}_{3} & -\mathrm{r}_{2} & \mathrm{r}_{1} & \mathrm{r}_{0}
\end{array}\right)
$$

The norm of $\mathrm{q}=\mathrm{q}_{0}+\mathrm{q}_{1} \mathbf{i}+\mathrm{q}_{2} \mathbf{j}+\mathrm{q}_{3} \mathbf{k}$ is

$$
N_{\mathrm{q}}=\mathrm{q} \overline{\mathrm{q}}=\overline{\mathrm{q}} \mathrm{q}=\mathrm{q}_{0}^{2}-\mathrm{q}_{1}^{2}
$$

and its modulus is

$$
\|\mathrm{q}\|=\sqrt{\left|N_{\mathrm{q}}\right|}=\sqrt{\left|\mathrm{q}_{0}^{2}-\mathrm{q}_{1}^{2}\right|} .
$$

Split semi-quaternions with $N_{\mathrm{q}}= \pm 1$ are called unit.

The multiplicative-inverse of a split semi-quaternion $\mathrm{q}=\mathrm{q}_{0}+\mathrm{q}_{1} \mathbf{i}+\mathrm{q}_{2} \mathbf{j}+\mathrm{q}_{3} \mathbf{k}$ is defined to be

$$
\mathrm{q}^{-1}=\frac{\overline{\mathrm{q}}}{N_{\mathrm{q}}}
$$

provided $N_{\mathrm{q}} \neq 0$, i.e. $\mathrm{q}_{0} \neq \pm \mathrm{q}_{1}$. Thus, a non-zero split semi-quaternion $\mathrm{p}=\mathrm{p}_{0}+\mathrm{p}_{1} \mathbf{i}+\mathrm{p}_{2} \mathbf{j}+\mathrm{p}_{3} \mathrm{k}$ provided $\mathrm{p}_{0}= \pm \mathrm{p}_{1}$ does not have an inverse. If $\mathrm{q}$ has an inverse, then $\mathrm{qq}^{-1}=\mathrm{q}^{-1} \mathrm{q}=1$. Unlike real-quaternion algebra, algebra of split semi-quaternions does not forms a division algebra. For more information about split semiquaternions see [13].

A unit split semi-quaternion $q=q_{0}+q_{1} \mathbf{i}+q_{2} \mathbf{j}+q_{3} \mathbf{k}$ can also be expressed by one of the following two matrices:

1. If $\mathrm{q}_{0}^{2}-\mathrm{q}_{1}^{2}=1$, then

$$
\mathrm{Q}_{1}:=\left(\begin{array}{cccc}
\cosh \alpha & \sinh \alpha & 0 & 0 \\
\sinh \alpha & \cosh \alpha & 0 & 0 \\
\mathrm{q}_{2} & -\mathrm{q}_{3} & \cosh \alpha & \sinh \alpha \\
\mathrm{q}_{3} & -\mathrm{q}_{2} & \sinh \alpha & \cosh \alpha
\end{array}\right)
$$

2. If $\mathrm{q}_{0}^{2}-\mathrm{q}_{1}^{2}=-1$, then

$$
\mathrm{Q}_{2}:=\left(\begin{array}{cccc}
\sinh \alpha & \cosh \alpha & 0 & 0 \\
\cosh \alpha & \sinh \alpha & 0 & 0 \\
\mathrm{q}_{2} & -\mathrm{q}_{3} & \sinh \alpha & \cosh \alpha \\
\mathrm{q}_{3} & -\mathrm{q}_{2} & \cosh \alpha & \sinh \alpha
\end{array}\right)
$$


where $\alpha \in \mathbb{R}$. Since $\varepsilon=\mathrm{Q}_{1}^{\mathrm{t}} \varepsilon \mathrm{Q}_{1}$ (resp. $\left.\varepsilon=\mathrm{Q}_{2}^{\mathrm{t}} \varepsilon \mathrm{Q}_{2}\right)$ and $\operatorname{det} \mathrm{Q}_{1}=\cosh ^{2} \alpha-\sinh ^{2} \alpha=1\left(\right.$ resp. $\operatorname{det} \mathrm{Q}_{2}=\sinh ^{2} \alpha-$ $\cosh ^{2} \alpha=-1$ ), the matrix $Q_{1}$ (resp. $Q_{2}$ ) is semi-orthogonal for the metric tensor

$$
\varepsilon:=\left(\begin{array}{cccc}
1 & 0 & 0 & 0 \\
0 & -1 & 0 & 0 \\
0 & 0 & 0 & 0 \\
0 & 0 & 0 & 0
\end{array}\right)
$$

Here $Q_{1}^{t}\left(\right.$ resp. $\left.Q_{2}^{t}\right)$ is the transpose of $Q_{1}\left(\right.$ resp. $Q_{2}$ ). Therefore, unit split semi-quaternions can be used to represent rotations or reflections, see [7].

Let us denote the set of all unit split semi-quaternions (i.e., unit split semi 3-sphere) by $T \mathbb{R}_{1}^{2}$. A unit split semi-quaternion $\mathrm{q}=\mathrm{q}_{0}+\mathrm{q}_{1} \mathbf{i}+\mathrm{q}_{2} \mathbf{j}+\mathrm{q}_{3} \mathbf{k}$ can be written in two parts $\mathrm{q}^{\bullet}=\left(\mathrm{q}_{0}, \mathrm{q}_{1}\right) \in S_{1}^{1}$ and $\mathrm{q}^{\star}=\left(\mathrm{q}_{2}, \mathrm{q}_{3}\right) \in \mathbb{R}_{1}^{2}$ as $\mathrm{q}=\left(\mathrm{q}^{\bullet}, \mathrm{q}^{\star}\right) \in S_{1}^{1} \times \mathbb{R}_{1}^{2} \cong T \mathbb{R}_{1}^{2}$, where $S_{1}^{1}$ denotes the Lorentzian unit circle in semi-Euclidean plane $\mathbb{R}_{1}^{2}$ (that is, $\mathrm{q}_{0}^{2}-\mathrm{q}_{1}^{2}= \pm 1$ ) and $S_{1}^{1} \times \mathbb{R}_{1}^{2}$ a unit tangent bundle of $\mathbb{R}_{1}^{2}$, see Fig. 1 for the case $\mathrm{q}_{0}^{2}-\mathrm{q}_{1}^{2}=1$.

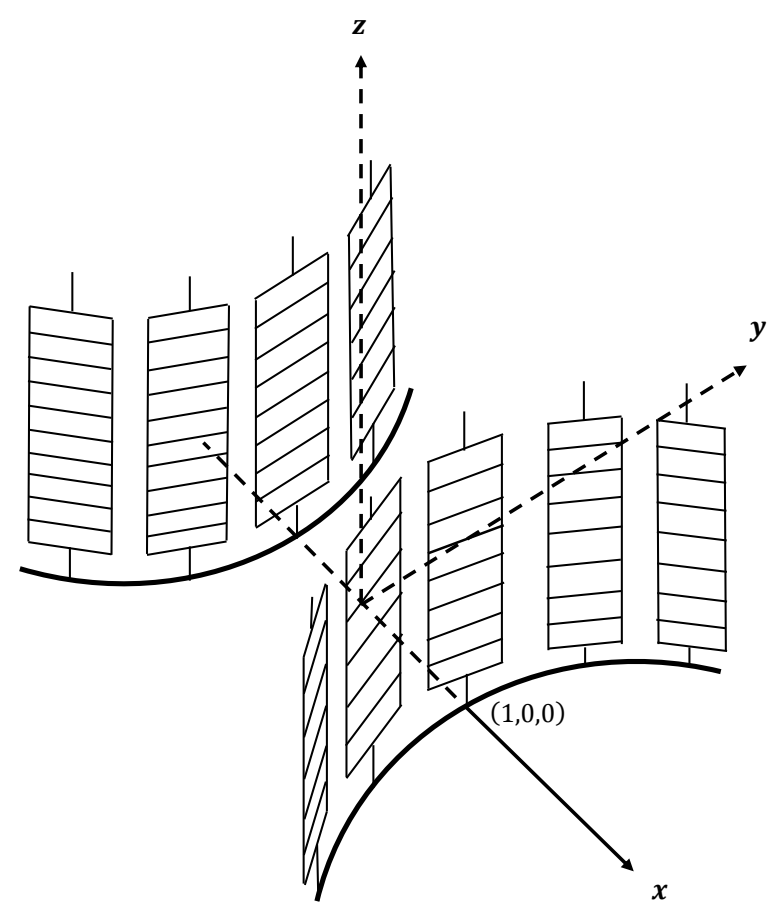

Figure 1. Unit split semi 3-sphere $T \mathbb{R}_{1}^{2}\left(\cong S_{1}^{1} \times \mathbb{R}_{1}^{2}\right)$.

Multiplication on $\mathbb{H}_{S}$ is a binary operation on $T \mathbb{R}_{1}^{2}$ and is associative, has the identity element $e=1+0 \mathbf{i}+$ $0 \mathbf{j}+0 \mathbf{k}$, and has the multiplicative-inverse $\mathrm{q}^{-1}= \pm \overline{\mathrm{q}}$ for each unit split semi-quaternion q. So, $T \mathbb{R}_{1}^{2}$ is a group with the multiplication operation on $\mathbb{H}_{s}$.

The algebra of split semi-quaternions $\mathbb{H}_{S}$ is isomorphic to the Clifford algebra $C \ell_{1,0,1}$ spanned by the basis $\left\{1, e_{1}, e_{2}, e_{1} e_{2}=e_{3}\right\}$ of a degenerate quadratic form with degeneracy in 1-dimension by identifying the quaternionic units $\mathbf{i}, \mathbf{j}, \mathbf{k}$ with $e_{1}, e_{2}, e_{3}$ in $C \ell_{1,0,1}$, respectively. The standard anti-commuting generators $e_{1}, e_{2}$ satisfy

$$
\left(e_{1}\right)^{2}=+1,\left(e_{2}\right)^{2}=\left(e_{3}\right)^{2}=0 \text { and } e_{1} e_{2}=-e_{2} e_{1} .
$$

\section{Lie Algebra of Unit Split Semi 3-Sphere $T \mathbb{R}_{1}^{2}$}

In this section, the Lie algebra of unit semi 3-sphere $T \mathbb{R}_{1}^{2}$ is considered. Initially, let us define the differentiable mapping

$$
\psi: \mathbb{H}_{\mathrm{S}} \rightarrow \mathbb{R} ; \quad \psi(\mathrm{q})=\mathrm{q}_{0}^{2}-\mathrm{q}_{1}^{2}
$$


for $\mathrm{q}=\mathrm{q}_{0}+\mathrm{q}_{1} \mathbf{i}+\mathrm{q}_{2} \mathbf{j}+\mathrm{q}_{3} \mathbf{k} \in \mathbb{H}_{\mathrm{S}}$. By restricting the domain set to $T \mathbb{R}_{1}^{2}$, we get the regular value of $\psi$ as 1 . The Jacobian matrix of $\psi$ is

$$
J_{\mathrm{q}}(\psi)=\left(\begin{array}{cccc}
2 \mathrm{q}_{0} & -2 \mathrm{q}_{1} & 0 & 0
\end{array}\right),
$$

and thus $\operatorname{rank}(\psi)=1$ (i.e., $\psi$ is surjective) for all $\mathrm{q} \in \psi^{-1}(1)=T \mathbb{R}_{1}^{2}$. So, $T \mathbb{R}_{1}^{2}$ is a submanifold of $\mathbb{H}_{\mathrm{S}}$. Moreover, since the mappings

$$
\alpha: T \mathbb{R}_{1}^{2} \times T \mathbb{R}_{1}^{2} \rightarrow T \mathbb{R}_{1}^{2} ; \quad \alpha(\mathrm{q}, \mathrm{p})=\mathrm{qp}
$$

and

$$
\beta: T \mathbb{R}_{1}^{2} \rightarrow T \mathbb{R}_{1}^{2} ; \quad \beta(\mathrm{q})=\mathrm{q}^{-1}= \pm \overline{\mathrm{q}}
$$

are differentiable, $T \mathbb{R}_{1}^{2}$ is a Lie group of dimension three.

The angular velocity matrix of a unit split semi-quaternion $\mathrm{q}_{=}=\mathrm{q}_{0}+\mathrm{q}_{1} \mathbf{i}+\mathrm{q}_{2} \mathbf{j}+\mathrm{q}_{3} \mathbf{k}$ can be given by one of the following two matrices:

1. If $\mathrm{q}_{0}^{2}-\mathrm{q}_{1}^{2}=1$, using Eq.(2.4), we get

$$
\mathcal{W}_{1}=\dot{\mathrm{Q}}_{1} \mathrm{Q}_{1}{ }^{-1}:=\left(\begin{array}{rrrr}
0 & 1 & 0 & 0 \\
1 & 0 & 0 & 0 \\
k & l & 0 & 1 \\
-l & -k & 1 & 0
\end{array}\right)
$$

2. If $\mathrm{q}_{0}^{2}-\mathrm{q}_{1}^{2}=-1$, using Eq.(2.5), we get

$$
\mathcal{W}_{2}=\dot{\mathrm{Q}}_{2} \mathrm{Q}_{2}{ }^{-1}:=\left(\begin{array}{rrrr}
0 & 1 & 0 & 0 \\
1 & 0 & 0 & 0 \\
l & k & 0 & 1 \\
-k & -l & 1 & 0
\end{array}\right)
$$

where in both

$$
\begin{aligned}
& k=\left(\dot{\mathrm{q}}_{2}-\mathrm{q}_{3}\right) \cosh \alpha+\left(\dot{\mathrm{q}}_{3}-\mathrm{q}_{2}\right) \sinh \alpha, \\
& l=\left(\mathrm{q}_{2}-\dot{\mathrm{q}}_{3}\right) \cosh \alpha+\left(\mathrm{q}_{3}-\dot{\mathrm{q}}_{2}\right) \sinh \alpha .
\end{aligned}
$$

Here, $\mathrm{Q}_{1}^{-1}$ and $\mathrm{Q}_{2}^{-1}$ are, respectively, the inverse matrices of $\mathrm{Q}_{1}$ and $\mathrm{Q}_{2}$, and $\dot{\mathrm{Q}}_{1}, \dot{\mathrm{Q}}_{2}, \dot{\mathrm{q}}_{2}, \dot{\mathrm{q}}_{3}$ are, respectively, the derivatives of $\mathrm{Q}_{1}, \mathrm{Q}_{2}, \mathrm{q}_{2}, \mathrm{q}_{3}$.

The Lie algebra of the group $T \mathbb{R}_{1}^{2}$ can also be given by one of the following two vector spaces:

1. Using Eq.(3.1), we have

$$
T_{1} \mathbb{R}_{1}^{2}=\operatorname{span}\left\{\lambda \mathcal{W}_{1}: \lambda \in \mathbb{R}\right\}
$$

2. Using Eq.(3.2), we haven

$$
T_{2} \mathbb{R}_{1}^{2}=\operatorname{span}\left\{\lambda \mathcal{W}_{2}: \lambda \in \mathbb{R}\right\}
$$

both equipped with the Lie product operation

$$
[\mathrm{Q}, \mathrm{P}]=\mathrm{QP}-\mathrm{PQ}
$$

where $\mathrm{Q}$ and $\mathrm{P}$ denotes, respectively, the matrix representations of any two unit split semi-quaternions $\mathrm{q}$ and p with $N_{\mathrm{q}}=N_{\mathrm{p}}= \pm 1$, and QP denotes the usual matrix product of Q and P.

We can write

$$
\lambda \mathcal{W}_{1}:=\lambda\left(\begin{array}{llll}
0 & 1 & 0 & 0 \\
1 & 0 & 0 & 0 \\
0 & 0 & 0 & 1 \\
0 & 0 & 1 & 0
\end{array}\right)+\lambda l\left(\begin{array}{rrrr}
0 & 0 & 0 & 0 \\
0 & 0 & 0 & 0 \\
1 & 0 & 0 & 0 \\
0 & -1 & 0 & 0
\end{array}\right)+\lambda k\left(\begin{array}{rrrr}
0 & 0 & 0 & 0 \\
0 & 0 & 0 & 0 \\
0 & 1 & 0 & 0 \\
-1 & 0 & 0 & 0
\end{array}\right)
$$


and

$$
\lambda \mathcal{W}_{2}:=\lambda\left(\begin{array}{llll}
0 & 1 & 0 & 0 \\
1 & 0 & 0 & 0 \\
0 & 0 & 0 & 1 \\
0 & 0 & 1 & 0
\end{array}\right)+\lambda k\left(\begin{array}{rrrr}
0 & 0 & 0 & 0 \\
0 & 0 & 0 & 0 \\
1 & 0 & 0 & 0 \\
0 & -1 & 0 & 0
\end{array}\right)+\lambda l\left(\begin{array}{rrrr}
0 & 0 & 0 & 0 \\
0 & 0 & 0 & 0 \\
0 & 1 & 0 & 0 \\
-1 & 0 & 0 & 0
\end{array}\right)
$$

This means that the basis elements both of the vector spaces $T_{1} \mathbb{R}_{1}^{2}$ and $T_{2} \mathbb{R}_{1}^{2}$ are

$$
\mathbf{i}:=\left(\begin{array}{llll}
0 & 1 & 0 & 0 \\
1 & 0 & 0 & 0 \\
0 & 0 & 0 & 1 \\
0 & 0 & 1 & 0
\end{array}\right), \mathbf{j}:=\left(\begin{array}{rrrr}
0 & 0 & 0 & 0 \\
0 & 0 & 0 & 0 \\
1 & 0 & 0 & 0 \\
0 & -1 & 0 & 0
\end{array}\right), \mathbf{k}:=\left(\begin{array}{rrrr}
0 & 0 & 0 & 0 \\
0 & 0 & 0 & 0 \\
0 & 1 & 0 & 0 \\
-1 & 0 & 0 & 0
\end{array}\right)
$$

satisfying

$$
[\mathbf{i}, \mathbf{j}]=-2 \mathbf{k}, \quad[\mathbf{j}, \mathbf{k}]=0, \quad[\mathbf{k}, \mathbf{i}]=2 \mathbf{j} .
$$

Left invariant vector fields on $T \mathbb{R}_{1}^{2}$ at the point

$$
\mathrm{q}=\mathrm{q}_{0}+\mathrm{q}_{1} \mathbf{i}+\mathrm{q}_{2} \mathbf{j}+\mathrm{q}_{3} \mathbf{k}
$$

are $X=\mathrm{q} \mathbf{i}, Y=\mathrm{q} \mathbf{j}$ and $Z=\mathrm{q} \mathbf{k}$. These vector fields can be expressed as

$$
\begin{aligned}
X & =\mathrm{q}_{1}+\mathrm{q}_{0} \mathbf{i}-\mathrm{q}_{3} \mathbf{j}-\mathrm{q}_{2} \mathbf{k}, \\
Y & =\mathrm{q}_{0} \mathbf{j}+\mathrm{q}_{1} \mathbf{k}, \\
Z & =\mathrm{q}_{1} \mathbf{j}+\mathrm{q}_{0} \mathbf{k}
\end{aligned}
$$

when we restrict ourselves to the unit element $e=1+0 \mathbf{i}+0 \mathbf{j}+0 \mathbf{k}$ of the Lie group $T \mathbb{R}_{1}^{2}$.

Let us denote the Lie algebra of the group $T \mathbb{R}_{1}^{2}$ by $T_{*} \mathbb{R}_{1}^{2}$, which is given by $T_{1} \mathbb{R}_{1}^{2}$ for the case $\mathrm{q}_{0}^{2}-\mathrm{q}_{1}^{2}=1$, see Eq.(3.3), and by $T_{2} \mathbb{R}_{1}^{2}$ for the case $\mathrm{q}_{0}^{2}-\mathrm{q}_{1}^{2}=-1$, see Eq.(3.4). We denote the matrix representation of the Lie algebra $T_{*} \mathbb{R}_{1}^{2}$ by

$$
\operatorname{Ad} X: T_{*} \mathbb{R}_{1}^{2} \rightarrow T_{*} \mathbb{R}_{1}^{2} ; \quad \operatorname{Ad} X(Y)=[Y, X]
$$

where $X, Y \in T_{*} \mathbb{R}_{1}^{2}$. The form $K(X, Y)=\operatorname{Tr}(\operatorname{Ad} X \circ \operatorname{Ad} Y)$ for all $Y, Y \in T_{*} \mathbb{R}_{1}^{2}$ is the Killing bilinear form on $X \in T_{*} \mathbb{R}_{1}^{2}$. Here, $\operatorname{Tr}(\operatorname{Ad} X \circ \operatorname{Ad} Y)$ stands for the trace of the mapping

$$
\operatorname{Ad} X \circ \operatorname{Ad} Y: T_{*} \mathbb{R}_{1}^{2} \rightarrow T_{*} \mathbb{R}_{1}^{2} ; \quad \operatorname{Ad} X \circ \operatorname{Ad} Y(Z)=[X,[Y, Z]]
$$

where " $\circ$ " deontes the usual matrix product.

Let $X:=\mathbf{x}=\mathrm{x}_{1} \mathbf{i}+\mathrm{x}_{2} \mathbf{j}+\mathrm{x}_{3} \mathbf{k}$ and $Y:=\mathbf{y}=\mathrm{y}_{1} \mathbf{i}+\mathrm{y}_{2} \mathbf{j}+\mathrm{y}_{3} \mathbf{k}$ be two vectors from $T_{*} \mathbb{R}_{1}^{2}$. The corresponding matrices of the mappings $\operatorname{Ad} X, \operatorname{Ad} Y$ and $\operatorname{Ad} X \circ \operatorname{Ad} Y$ are

$$
\operatorname{Ad} X:=\left(\begin{array}{ccc}
0 & 0 & 0 \\
2 \mathrm{x}_{3} & 0 & -2 \mathrm{x}_{1} \\
2 \mathrm{x}_{2} & -2 \mathrm{x}_{1} & 0
\end{array}\right) ; \operatorname{Ad} Y:=\left(\begin{array}{ccc}
0 & 0 & 0 \\
2 \mathrm{y}_{3} & 0 & -2 \mathrm{y}_{1} \\
2 \mathrm{y}_{2} & -2 \mathrm{y}_{1} & 0
\end{array}\right)
$$

and

$$
\operatorname{Ad} X \circ \operatorname{Ad} Y:=\left(\begin{array}{ccc}
0 & 0 & 0 \\
-4 \mathrm{x}_{1} \mathrm{y}_{2} & 4 \mathrm{x}_{1} \mathrm{y}_{1} & 0 \\
-4 \mathrm{x}_{1} \mathrm{y}_{3} & 0 & 4 \mathrm{x}_{1} \mathrm{y}_{1}
\end{array}\right)
$$

We get

$$
K(X, Y)=\operatorname{Tr}(\operatorname{Ad} X \circ \operatorname{Ad} Y)=-8\langle X, Y\rangle^{*}=8 \mathrm{x}_{1} \mathrm{y}_{1} .
$$

If the first component $\mathrm{x}_{1} \in \mathbb{R}$ of $X$ is non-zero, then

$$
K(X, X)=-8\langle X, X\rangle^{*}=8 \mathrm{x}_{1}^{2}>0
$$

and this means that the set

$$
\widetilde{T} \mathbb{R}_{1}^{2}=\left\{X=\mathrm{x}_{1} \mathbf{i}+\mathrm{x}_{2} \mathbf{j}+\mathrm{x}_{3} \mathbf{k}: \mathrm{x}_{1}, \mathrm{x}_{2}, \mathrm{x}_{2} \in \mathbb{R} \text { and } \mathrm{x}_{1} \neq 0\right\}
$$

is a non-compact Lie group. 


\section{Planar Rotation and Split Semi-Quaternions}

A motion $f_{\alpha}$ of the semi-Euclidean plane $\mathbb{R}_{1}^{2}$ can be given with respect to an orthonormal coordinate system as

$$
f_{\alpha}: \mathbb{R}_{1}^{2} \rightarrow \mathbb{R}_{1}^{2} ; \quad \mathbf{x} \mapsto A_{\alpha} \cdot \mathbf{x}+\mathbf{c}
$$

where

$$
A_{\alpha}=\left(\begin{array}{cc}
\cosh \alpha & \sinh \alpha \\
\sinh \alpha & \cosh \alpha
\end{array}\right) .
$$

Here, $A_{\alpha}$ is a semi-orthogonal matrix and represents a pseudo-rotation (also known as boost) of $\mathbb{R}_{1}^{2}$ through a positive oriented hyperbolic angle $\alpha \in \mathbb{R}$, and the vector $\mathbf{c}=\left(c_{1}, c_{2}\right) \in \mathbb{R}_{1}^{2}$ is a $2 \times 1$ column matrix representing the translation part of the motion, see [16, 10].

Lemma 4.1 ([7], Proposition 3.1). The non-pure unit split semi-quaternion

$$
\mathrm{q}=-\cosh \frac{\alpha}{2}+\sinh \frac{\alpha}{2} \mathbf{i}+\mathrm{q}_{2} \mathbf{j}+\mathrm{q}_{3} \mathbf{k} ; \quad \sinh \frac{\alpha}{2} \neq 0
$$

represents a negative oriented pseudo-rotation in $\mathbb{R}_{1}^{2}$ with an hyperbolic angle $\alpha \in \mathbb{R}$ and center

$$
\mathrm{m}=\left(\frac{\mathrm{q}_{2}}{\sinh \frac{\alpha}{2}}, \frac{\mathrm{q}_{3}}{\sinh \frac{\alpha}{2}}\right) .
$$

Example 4.1. Let $\mathrm{q}=\mathrm{q}_{0}+\mathrm{q}_{1} \mathbf{i}+\mathrm{q}_{2} \mathbf{j}+\mathrm{q}_{3} \mathbf{k}$ be a non-pure unit split semi-quaternion defined by

$$
\mathrm{q}_{0}=-\cosh \frac{\pi}{8} ; \quad \mathrm{q}_{1}=\sinh \frac{\pi}{8} ; \quad \mathrm{q}_{2}=1 ; \quad \mathrm{q}_{3}=-\frac{3}{5} .
$$

Then, the map

$$
f_{\mathrm{q}}:\left(\begin{array}{l}
x \\
y
\end{array}\right) \mapsto\left(\begin{array}{cc}
\mathrm{q}_{0}^{2}+\mathrm{q}_{1}^{2} & 2 \mathrm{q}_{0} \mathrm{q}_{1} \\
2 \mathrm{q}_{0} \mathrm{q}_{1} & \mathrm{q}_{0}^{2}+\mathrm{q}_{1}^{2}
\end{array}\right)\left(\begin{array}{c}
x \\
y
\end{array}\right)+\left(\begin{array}{c}
-2\left(\mathrm{q}_{1} \mathrm{q}_{2}+\mathrm{q}_{0} \mathrm{q}_{3}\right) \\
-2\left(\mathrm{q}_{1} \mathrm{q}_{3}+\mathrm{q}_{0} \mathrm{q}_{2}\right)
\end{array}\right)
$$

represents a semi-Euclidean planar motion because

$$
\operatorname{det}\left(\begin{array}{cc}
q_{0}^{2}+q_{1}^{2} & 2 q_{0} q_{1} \\
2 q_{0} q_{1} & q_{0}^{2}+q_{1}^{2}
\end{array}\right)=1 .
$$

Moreover, we obtain

$$
f_{\mathrm{q}}\left(\frac{1}{\sinh \frac{\pi}{8}}, \frac{-3}{5 \sinh \frac{\pi}{8}}\right)=\left(\frac{1}{\sinh \frac{\pi}{8}}, \frac{-3}{5 \sinh \frac{\pi}{8}}\right) .
$$

Thus, the map $f_{\mathrm{q}}$ represents a negative oriented pseudo-rotation in $\mathbb{R}_{1}^{2}$ with an hyperbolic angle $\pi / 4 \in \mathbb{R}$ and center

$$
\mathrm{m}=\left(\frac{1}{\sinh \frac{\pi}{8}}, \frac{-3}{5 \sinh \frac{\pi}{8}}\right) .
$$

The pseudo-rotation in semi-Euclidean plane $\mathbb{R}_{1}^{2}$, which is given by Lem. 4. 1., is obtained using non-pure unit split semi-quaternions. However, by the following Prop. 4. 2, we show that if we use pure unit split semi-quaternions, we obtain the rotations in Euclidean plane $\mathbb{R}^{2}$.

Proposition 4.1. Let $\boldsymbol{p}=\varepsilon_{1} \mathbf{i}+\mathrm{p}_{2} \mathbf{j}+\mathrm{p}_{3} \mathbf{k}$ and $\boldsymbol{q}=\varepsilon_{2} \mathbf{i}+\mathrm{q}_{2} \mathbf{j}+\mathrm{q}_{3} \mathbf{k}$ be unit pure split semi-quaternions for $\varepsilon_{1}= \pm 1$ and $\varepsilon_{2}= \pm 1$. Then,

$$
\boldsymbol{p} \boldsymbol{q} \boldsymbol{p}^{-1}=\boldsymbol{p} \boldsymbol{q} \boldsymbol{p}=\boldsymbol{q}^{\prime}=\varepsilon_{2} \mathbf{i}+\mathrm{q}_{2}^{\prime} \mathbf{j}+\mathrm{q}_{3}^{\prime} \mathbf{k}:=\left(\mathrm{q}_{2}^{\prime}, \mathrm{q}_{3}^{\prime}\right) \in \mathbb{R}^{2}
$$

corresponds to the reflection of the point $\mathrm{q}=\left(\mathrm{q}_{2}, \mathrm{q}_{3}\right) \in \mathbb{R}^{2}$ in the point $\mathrm{m}=\left(\varepsilon_{1} \varepsilon_{2} \mathrm{p}_{2}, \varepsilon_{1} \varepsilon_{2} \mathrm{p}_{3}\right) \in \mathbb{R}^{2}$ in Euclidean plane $\mathbb{R}^{2}$.

Proof. Let $\mathbf{p}=\varepsilon_{1} \mathbf{i}+\mathrm{p}_{2} \mathbf{j}+\mathrm{p}_{3} \mathbf{k}$ and $\mathbf{q}=\varepsilon_{2} \mathbf{i}+\mathrm{q}_{2} \mathbf{j}+\mathrm{q}_{3} \mathbf{k}$ be unit pure split semi-quaternions for $\varepsilon_{1}= \pm 1$ and $\varepsilon_{2}= \pm 1$. Then,

We can define a linear map

$$
\mathbf{q}^{\prime}=\mathbf{p q p}^{-1}=\mathbf{p q p}=\varepsilon_{2} \mathbf{i}+\mathrm{q}_{2}^{\prime} \mathbf{j}+\mathrm{q}_{3}^{\prime} \mathbf{k} .
$$

$$
\varphi_{p}: \mathbb{R}^{2} \rightarrow \mathbb{R}^{2}
$$


as

$$
\left(\begin{array}{c}
\mathrm{q}_{2} \\
\mathrm{q}_{3}
\end{array}\right) \mapsto\left(\begin{array}{rr}
-1 & 0 \\
0 & -1
\end{array}\right)\left(\begin{array}{l}
\mathrm{q}_{2} \\
\mathrm{q}_{3}
\end{array}\right)+\left(\begin{array}{c}
2 \varepsilon_{1} \varepsilon_{2} \mathrm{p}_{2} \\
2 \varepsilon_{1} \varepsilon_{2} \mathrm{p}_{3}
\end{array}\right)=\left(\begin{array}{l}
\mathrm{q}_{2}^{\prime} \\
\mathrm{q}_{3}^{\prime}
\end{array}\right)
$$

It is straightforward that

$$
\varphi_{p}\left(\varepsilon_{1} \varepsilon_{2} \mathrm{p}_{2}, \varepsilon_{1} \varepsilon_{2} \mathrm{p}_{3}\right)=\left(\varepsilon_{1} \varepsilon_{2} \mathrm{p}_{2}, \varepsilon_{1} \varepsilon_{2} \mathrm{p}_{3}\right)
$$

and this means that the linear map $\varphi_{p}$ can be used to represent a rotation with an angle $180^{\circ}$ and center $\mathrm{m}=\left(\varepsilon_{1} \varepsilon_{2} \mathrm{p}_{2}, \varepsilon_{1} \varepsilon_{2} \mathrm{p}_{3}\right)$ (i.e., equivalent to a reflection in the point $\mathrm{m}$ ) in Euclidean plane $\mathbb{R}^{2}$.

Example 4.2. Let us consider the unit pure split semi-quaternions $\mathbf{p}=-\mathbf{i}+\mathbf{j}-\mathbf{k}$ and $\mathbf{q}=\mathbf{i}-2 \mathbf{j}+3 \mathbf{k}$. Then, it can be easily observed that the product

$$
\mathbf{p q p}^{-1}=\mathbf{p q p}=\mathbf{i}+0 \mathbf{j}-\mathbf{k}:=(0,-1) \in \mathbb{R}^{2}
$$

represents a reflection of the point $\mathrm{q}=\left(\mathrm{q}_{2}, \mathrm{q}_{3}\right)=(-2,3) \in \mathbb{R}^{2}$ in the point $\mathrm{m}=\left(\varepsilon_{1} \varepsilon_{2} \mathrm{p}_{2}, \varepsilon_{1} \varepsilon_{2} \mathrm{p}_{3}\right)=(-1,1) \in \mathbb{R}^{2}$ in Euclidean plane $\mathbb{R}^{2}$.

\section{Conclusions}

The algebra of real-quaternions can be considered by representing rotations and reflections in Euclidean plane $\mathbb{R}^{2}$, see [9]. The algebra of split semi-quaternions can be considered by representing pseudo-rotations in $\mathbb{R}_{1}^{2}$ and planar rotations in $\mathbb{R}^{2}$. Moreover, we can consider the set of all unit split semi-quaternions (i.e., unit split semi 3-sphere) as a unit tangent bundle of $\mathbb{R}_{1}^{2}$.

\section{References}

[1] Abłamowicz, R. and Sobczyk, G., Lectures on Clifford (Geometric) Algebras and Applications. Birkhäuser, Boston, 2004.

[2] Abłamowicz, R., Computations with Clifford and Grassmann Algebras, Adv. Appl. Clifford Algebras, 19 (2009), 499-545.

[3] Abłamowicz, R. and Fauser, B., On the Transposition Anti-Involution in Real Clifford Algebras I: The Transposition Map, Linear Multilinear A, 59 (2011), 1313-1358.

[4] Abłamowicz, R. and Fauser, B., On the Transposition Anti-Involution in Real Clifford Algebras II: Stabilizer Groups of Primitive Idempotents, Linear Multilinear A, 59 (2011), 1359-1381.

[5] Abłamowicz, R. and Fauser, B., On the Transposition Anti-Involution in Real Clifford Algebras III: The Automorphism Group of the Transposition Scalar Product on Spinor Spaces, Linear Multilinear A, 60 (2012), 621-644.

[6] Aslan, S. and Yayli, Y., Canal Surfaces with Quaternions, Adv. Appl. Clifford Algebras, 26 (2016), 31-38.

[7] Bekar, M. and Yayli, Y., Semi-Euclidean Quasi-Elliptic Planar Motion, Int. J. Geom. Methods Mod. Phys., 13 (2016), 1650089 (11 pages), DOI: 10.1142/S0219887816500894.

[8] Bekar, M. and Yayli, Y., Lie Aigebra of Unit Tangent Bundle, Adv. Appl. Clifford Algebras, 27 (2017), 965-975.

[9] Ell, T. A. and Sangwine, S. J., Quaternion Involutions and Anti-Involutions, Comput. Math. Appl., 53 (2007), 137-143.

[10] Es, H., First and Second Acceleration Poles in Lorentzian Homothetic Motions, Commun. Fac. Sci. Univ. Ank. Ser. A 1 Math. Stat., 67 (2018), $19-28$.

[11] Hahn, A. J., Quadratic Algebras, Clifford Algebras and Arithmetic Witt Groups, Springer-Verlag, New York, 1994.

[12] Hamilton, W. R., On a New Species of Imaginary Quantities Connected with the Theory of Quaternions, P. Roy. Irish Academy, 2 (1844), 424-434.

[13] Jafari, M., Split Semi-Quaternions Algebra in Semi-Euclidean 4-Space, Cumhuriyet Science Journal, 3636 (2015), 70-77.

[14] Kuipers, J. B., Quaternions and Rotation Sequences. Princeton University Press, New Jersey, 1999.

[15] Kula, L. and Yayli, Y., Split Quaternions and Rotations in Semi-Euclidean Space E E, J. Korean Math. Soc., 6 (2007), $1313-1327$.

[16] Lopez, R., Differential Geometry of Curves and Surfaces in Lorentz-Minkowski Space, Int. Electron. J. Geom., 7 (2014), 44-107.

[17] Ward, J. P., Quaternions and Cayley Algebras and Applications, Kluwer Academic Publishers, Dordrecht, 1996.

\section{Affiliations}

MURAT BEKAR

AdDress: Gazi University, Faculty of Science and Letters, Department of Mathematics, 06900, Polatli/Ankara-Turkey

E-MAIL: murat-bekar@hotmail.com

ORCID ID: orcid.org/0000-0003-3617-6940

www.iejgeo.com 